\title{
NODAL SETS FOR SUMS OF EIGENFUNCTIONS ON RIEMANNIAN MANIFOLDS
}

\author{
HAROLD DONNELLY
}

(Communicated by Peter Li)

\begin{abstract}
ABSTRACr. Quantitative versions of unique continuation are proved for finite sums of eigenfunctions of the Laplacian on compact Riemannian manifolds. The results include a lower bound for the order of vanishing, a growth estimate for the supremum on compact balls, and a gradient bound. For real analytic metrics, an upper bound for the Hausdorff measure of the zero set is derived.
\end{abstract}

\section{INTRODUCTION}

Let $M^{d}$ be a compact Riemannian manifold. The associated Laplacian $\Delta$ is a second order elliptic operator acting on functions. Suppose that $\phi$ is an eigenfunction of $\Delta$ with eigenvalue $\lambda, \Delta \phi=-\lambda \phi$. In [3], we proved several theorems, concerning the nodal set of $\phi$, which quantify the unique continuation theorems of Aronszajn [1] and Cordes [2]. These include a lower bound for the order of vanishing of $\phi$, a growth estimate for the supremum of $\phi$ on compact balls, and a gradient bound. For real analytic metrics, an upper bound for the $(d-1)$-dimensiona! Hausdorff measure of the nodal set of $\phi$ is derived. Here $d$ is the dimension of $M$. These estimates have a precise dependence upon the eigenvalue $\lambda$.

The purpose of the present note is to generalize the results of [3] to finite sums of eigenfunctions $f=\sum_{i=1}^{n} \phi_{i}$, corresponding to eigenvalues $-\lambda_{i}, \lambda_{i} \leq \lambda$. Such a generalization is natural because our earlier work was motivated by the idea that an eigenfunction should behave like an ordinary polynomial or trigonometric polynomial. From this point of view, it seems most appropriate to study finite sums of eigenfunctions, rather than just individual eigenfunctions. Now one is concerned with dependence upon the two parameters $n$ and $\lambda$. For real analytic metrics, both $n$ and $\lambda$ may become arbitrarily large. In the $C^{\infty}$ case, our methods require that $n$ should remain bounded, while $\lambda$ approaches infinity. Many of the details of proof are similar to the previous study of single eigenfunctions.

Received by the editors October 22, 1992.

1991 Mathematics Subject Classification. Primary 58G03, 58G25. 


\section{DeRIVATIVES OF SUMS OF EIGENFUNCTIONS}

Suppose that $f$ lies in the kernel of the differential operator $\prod_{i=1}^{n}\left(\Delta+\lambda_{i}\right)$, where $0<\lambda_{i} \leq \lambda$ are distinct. In particular, $f$ might be a finite sum, $f=\sum_{i=1}^{n} \phi_{i}$, of eigenfunctions $\phi_{i}$ of the Laplacian $\Delta$, corresponding to the eigenvalues $-\lambda_{i}$. We would like to apply local elliptic theory, for $\prod_{i=1}^{n}\left(\Delta+\lambda_{i}\right)$, to bound the derivatives of $f$ in terms of the supremum norm of $f$. Since $n$ may become arbitrarily large, the coefficients of $\prod_{i=1}^{n}\left(\Delta+\lambda_{i}\right)$ involve many derivatives of the metric $g_{i j}$. Of course, there is no control for the arbitrarily high order dervatives of a $C^{\infty}$ function. Therefore, we assume that our metric $g_{i j}$ is real analytic. Those readers who are interested only in $C^{\infty}$ metrics may omit this section.

Our first task is to estimate the coefficients of $\Delta^{j}, 0<j \leq n$. We choose local analytic coordinates $x_{1}, x_{2}, \ldots, x_{d}$. By definition,

$$
\Delta=\frac{1}{\sqrt{g}} \frac{\partial}{\partial x_{i}}\left(\sqrt{g} g^{i j} \frac{\partial}{\partial x_{j}}\right)=\sum b_{i j} \frac{\partial^{2}}{\partial x_{i} \partial x_{j}}+\sum b_{k} \frac{\partial}{\partial x_{k}},
$$

where $b_{i j}=g^{i j}$ and $b_{k}=\frac{1}{\sqrt{g}} \frac{\partial}{\partial x_{i}}\left(g^{i k} \sqrt{g}\right)$. Note that $b_{i j}$ involves no derivatives of the metric, while $b_{k}$ involves one derivative of the metric. If $\alpha=$ $\left(\alpha_{1}, \alpha_{2}, \ldots, \alpha_{d}\right)$ is a multi-index, of order $|\alpha|=\alpha_{1}+\alpha_{2}+\cdots+\alpha_{d}$, the partial derivatives of a function $h$ will be given as

$$
h^{(\alpha)}=D^{\alpha} h=\frac{\partial^{\alpha_{1}+\alpha_{2}+\cdots+\alpha_{d}} h}{\partial x_{1}^{\alpha_{1}} \partial x_{2}^{\alpha_{2}} \cdots \partial x_{d}^{\alpha_{d}}} .
$$

Consider a monomial $m$ of the form $b_{i_{1} j_{1}}^{\left(\gamma_{1}\right)} b_{i_{2} j_{2}}^{\left(\gamma_{2}\right)} \cdots b_{i_{r} j_{r}}^{\left(\gamma_{r}\right)} b_{l_{1}}^{\left(\beta_{1}\right)} \cdots b_{l_{s}}^{\left(\beta_{s}\right)}$, where $\gamma_{i}$ and $\beta_{i}$ are multi-indices. We say that $m$ has type $r+s$ and total order $\left|\gamma_{1}\right|+\left|\gamma_{2}\right|+\cdots+\left|\gamma_{r}\right|+\left|\beta_{1}\right|+\left|\beta_{2}\right|+\cdots+\left|\beta_{s}\right|+s$ in the derivatives of the metric $g_{i j}$. Using this terminology, one may state:

Lemma 2.1. In a local analytic coordinate patch $\Delta^{j}=\sum a_{j, \alpha} D^{\alpha}$. The coefficients $a_{j, \alpha}$ are polynomials having at most $c_{1}^{j} j^{2 j}$ terms and each term is a monomial of type $j$ and total order $2 j-|\alpha|$, in the derivatives of the metric. Moreover, any derivative $D^{w} a_{j, \alpha}$ is a polynomial with no more than $c_{1}^{j} j^{2 j+|w|}$ terms and each term is a monomial of type $j$ and total order $2 j-|\alpha|+|w|$. Consequently, $\left|D^{w} a_{j, \alpha}\right| \leq c_{3}^{j+|w|}(j+|w|)^{4 j+|w|} j^{|w|}$, where $c_{3}$ depends upon the Taylor series of our real analytic metric $g_{i j}$.

Proof. For $j=1$, the lemma follows from the definitions. We proceed by induction on $j$. Now

$$
\begin{aligned}
\Delta^{j+1}= & \Delta \Delta^{j}=\left(\sum b_{i l} \frac{\partial^{2}}{\partial x_{i} \partial x_{l}}+\sum b_{k} \frac{\partial}{\partial x_{k}}\right)\left(\sum a_{j \alpha} D^{\alpha}\right) \\
= & \sum b_{i l} a_{j \alpha, i l} D^{\alpha}+\sum b_{i l} a_{j \alpha} \frac{\partial^{2}}{\partial x_{i} \partial x_{l}} D^{\alpha}+2 \sum b_{i l} a_{j \alpha, i} \frac{\partial}{\partial x_{l}} D^{\alpha} \\
& +\sum b_{k} a_{j \alpha, k} D^{\alpha}+\sum b_{k} a_{j \alpha} \frac{\partial}{\partial x_{k}} D^{\alpha} .
\end{aligned}
$$

Since each $b_{i l}$ has order zero and each $b_{k}$ has order one, it is clear that $a_{j+1, \beta}$ is of type $j+1$ and total order $2(j+1)-|\beta|$, in the derivatives of the metric. 
Moreover, differentiating a monomial of type $j$ creates a polynomial with $j$ terms, and we differentiate at most twice during each inductive step. So $a_{j+1, \beta}$ has at most $c_{1}^{j+1}(j+1)^{2(j+1)}$ terms.

Consider now the derivatives $D^{w} a_{j, \alpha}$ of the coefficients $a_{j, \alpha}$. The required characterization of $a_{j, \alpha}$ has been established, so we proceed by induction on $|w|$, with $j$ fixed. Each differentiation preserves the type and increases the order by one. Also, each differentiation multiplies the number of monomials by the fixed type $j$. This gives the necessary description of the derivatives $D^{w} a_{j, \alpha}$ of the coefficients.

To estimate $D^{w} a_{j, \alpha}$, we note that

$$
\left|D^{w} a_{j, \alpha}\right| \leq c_{1}^{j} j^{2 j+|w|} \max \left|b_{i_{1} k_{1}}^{\left(\gamma_{1}\right)} b_{i_{2} k_{2}}^{\left(\gamma_{2}\right)} \cdots b_{i_{r} k_{r}}^{\left(\gamma_{r}\right)} b_{l_{1}}^{\left(\beta_{1}\right)} \cdots b_{l_{s}}^{\left(\beta_{s}\right)}\right|,
$$

where the maximum is over all monomials of type $j$, and total order $2 j-|\alpha|+$ $|w|$. By analyticity of $b_{i k}$ and $b_{l}$ :

$$
\begin{aligned}
\left|D^{w} a_{j, \alpha}\right| & \leq c_{1}^{j} j^{j j+|w|} \max c_{2}^{\left|\gamma_{1}\right|+1} \gamma_{1} ! \cdots c_{2}^{\left|\gamma_{r}\right|+1} \gamma r ! c_{2}^{\left|\beta_{1}\right|+1} \beta_{1} ! \cdots c_{2}^{\left|\beta_{s}\right|+1} \beta_{s} ! \\
& \leq c_{1}^{j} c_{2}^{3 j+|w|} j^{2 j+|w|}(2 j+|w|) !
\end{aligned}
$$

The upper bound for $\left|D^{w} a_{j, \alpha}\right|$ now follows by Stirling's formula.

Using the expansion $\prod_{i=1}^{n} \Delta+\lambda_{i}=\sum \lambda_{i_{1}} \cdots \lambda_{i_{k}} \Delta^{n-k}$, we easily deduce

Lemma 2.2. The product $\prod_{i=1}^{n} \Delta+\lambda_{i}$ equals $\sum \bar{a}_{n, \alpha} D^{\alpha}$, where the derivatives of the coefficients $\bar{a}_{n, \alpha}$ satisfy the upper bound:

$$
\left|D^{w} \bar{a}_{n, \alpha}\right| \leq c_{4}^{n+|w|}(n+|w|)^{4 n+|w|} \lambda^{n-|\alpha| / 2} n^{|w|} .
$$

Proof. In $\prod_{i=1}^{n} \Delta+\lambda_{i}=\sum \lambda_{i_{1}} \lambda_{i_{2}} \cdots \lambda_{i_{k}} \Delta^{n-k}$, there are $2^{n}$ summands. The coefficient $\bar{a}_{n, \alpha}$ is also a sum of terms each involving at most $n-|\alpha| / 2$ factors $\lambda_{i} \leq \lambda$. Therefore $\left|D^{w} \bar{a}_{n, \alpha}\right| \leq 2^{n} \lambda^{n-|\alpha| / 2} \sum_{j=1}^{n}\left|D^{w} a_{j, \alpha}\right|$, where $a_{j, \alpha}$ is the coefficient of $D^{\alpha}$ in the power $\Delta^{j}$ of the Laplacian. By Lemma 2.1,

$$
\begin{aligned}
\left|D^{w} \bar{a}_{n, \alpha}\right| & \leq 2^{n} \lambda^{n-|\alpha| / 2} \sum_{j=1}^{n} c_{3}^{j+|w|} j^{|w|}(j+|w|)^{4 j+|w|} \\
& \leq n 2^{n} \lambda^{n-|\alpha| / 2} c_{3}^{n+|w|} n^{|w|}(n+|w|)^{4 n+|w|} .
\end{aligned}
$$

Lemma 2.2 follows easily.

On balls of sufficiently small size, we may now apply standard elliptic theory to bound the derivatives of functions $f$ lying in the kernel of $\prod_{i=1}^{n} \Delta+\lambda_{i}$. Our preparatory lemmas determine the scale size, so that the pullback of the operator to unit scale has bounded coefficients. The main result of this section is

Proposition 2.3. If $B_{1}$ is a ball of radius $\rho$ less than $\left(c_{5}^{n} \lambda n^{c_{6} n}\right)^{-1 / 2}$, then for $|\alpha|<2 n$, we have

$$
\sup _{B_{1}}\left|D^{\alpha} f\right| \leq c_{7}^{n|\alpha|} \rho^{-|\alpha|} n^{c_{8} n|\alpha|} \sup _{B_{2}}|f|,
$$

where $B_{2}$ is the double of $B_{1}$. In particular,

$$
\sup _{B_{1}}\left|D^{\alpha} f\right| \leq c_{7}^{2 n^{2}} \rho^{-|\alpha|} n^{c_{9} n^{2}} \sup _{B_{2}}|f| .
$$


Proof. By Lemma 2.2, the pullback of $\prod_{i=1}^{n} \Delta+\lambda_{i}$ to unit scale is of order $2 n$ with uniformly bounded coefficients, along with $c_{10} n$ of their derivatives. One applies the standard boot-strapping process, through Sobolev spaces, to the normalized operator. Each iteration, improving by one Sobolev index, brings a factor of magnitude, $c_{11}^{n} n^{c_{12} n}$ from the $2 n$th derivatives of a cutoff function on an annulus of radius $\left(c_{13} n\right)^{-1}$. One uses $|\alpha|+d / 2$ iterations and the Sobolev embedding theorem to bound $D^{\alpha} f$ in supremum norm. Rescaling gives the factor $\rho^{-|\alpha|}$.

\section{LOCAL PROPERTIES OF SUMS OF EIGENFUNCTIONS}

Suppose that $f=\sum_{i=1}^{n} \phi_{i}$ is a finite sum of eigenfunctions of the Laplacian $\Delta$, where $\phi_{i}$ corresponds to the eigenvalue $-\lambda_{i} \geq-\lambda$. More generally, simply assume that we have $\left(\prod_{i=1}^{n}\left(\Delta+\lambda_{i}\right)\right) f=0$. Let $\theta$ be a radially symmetric cutoff function, defined in a local coordinate system, satisfying $0 \leq \theta \leq 1$, and

$$
\theta(r)= \begin{cases}0, & r<\delta\left(1-(10 \beta)^{-1}\right), \\ 1, & \delta<r<h / 2 \\ 0, & r>2 h / 3\end{cases}
$$

Here $r$ is the distance, in the locally defined Euclidean sense, from the center of our coordinate patch. We will substitute $u=\theta f$ into a suitable Carleman inequality for $\prod_{i=1}^{n}\left(\Delta+\lambda_{i}\right)$. This gives estimates of the order of vanishing of $f$ and rate of growth of $f$, in terms of the parameters $n$ and $\lambda$. If the Riemannian metric underlying $\Delta$ is real analytic, then $n$ may become arbitrarily large, for the estimates below. For $C^{\infty}$ metrics, $n$ must remain less than a fixed constant. In either $C^{\infty}$ or the real analytic category, $\lambda$ may become arbitrarily large.

We will need upper bounds, for derivatives of arbitrary order, of our cutoff function $\theta$. Since $\theta$ is only $C^{\infty}$, but not real analytic, $\left|D^{\alpha} \theta\right|$ may grow faster than $|\alpha| ! \sim \sqrt{2 \pi}|\alpha|^{|\alpha|+1 / 2} e^{-|\alpha|}$, by Stirling's formula. It is an elementary exercise to derive a compatible upper estimate:

Lemma 3.1.

$$
\sup _{0<r<h / 2}\left|D^{\alpha} \theta\right| \leq c_{1}^{|\alpha|}|\alpha|^{c_{2}|\alpha|} \delta^{-|\alpha|} \beta^{|\alpha|}
$$

$$
\sup _{h / 2<r<\infty}\left|D^{\alpha} \theta\right| \leq c_{1}^{|\alpha|}|\alpha|^{c_{2}|\alpha|} h^{-|\alpha|}
$$

One combines this with the results of the previous section to deduce:

Lemma 3.2. If $\beta>a_{1} \sqrt{\lambda}+a_{2}$, then

$$
\begin{aligned}
& \left|\left(\prod_{i=1}^{n}\left(\Delta+\lambda_{i}\right)\right)(\theta f)\right|(x) \\
& \quad \leq \begin{cases}c_{1}^{n^{2}} n^{c_{2} n^{2}}(\delta / \beta)^{-2 n}\|f\|_{\infty}, & \delta\left(1-(10 \beta)^{-1}\right)<r<\delta, \\
c_{1}^{n^{2}} n^{c_{2} n^{2}} h^{-2 n} \lambda^{n}\|f\|_{\infty}, & h / 2<r<2 h / 3 .\end{cases}
\end{aligned}
$$


Here $\|f\|_{\infty}$ is taken on a ball $B\left(x,\left(c_{3}^{n} n^{c_{4} n}\right)^{-1 / 2} \delta / \beta\right)$, for $\delta\left(1-(10 \beta)^{-1}\right)<$ $r<\delta$, while $\|f\|_{\infty}$ is taken on $B\left(x,\left(c_{3}^{n} \lambda n^{c_{4} n}\right)^{-1 / 2} h\right)$, for $h / 2<r<2 h / 3$.

Proof. The coefficients of $\prod_{i=1}^{n}\left(\Delta+\lambda_{i}\right)$ were estimated in Lemma 2.2. Consequently, we have

$$
\begin{aligned}
\left|\left(\prod_{i=1}^{n} \Delta+\lambda_{i}\right)(\theta f)\right|(x) & =\left|\sum \bar{a}_{n, \alpha} D^{\alpha}(\theta f)(x)\right| \\
& \leq c_{5}^{n} n^{4 n} \sum_{|\alpha| \leq 2 n} \lambda^{n-|\alpha| / 2}\left|D^{\alpha}(\theta f)\right|(x) .
\end{aligned}
$$

Now, expanding out via Leibniz's formula, one gets for each $|\alpha| \leq 2 n$,

$$
\left|D^{\alpha}(\theta f)\right|(x) \leq c_{6}^{n} \sup _{|\varepsilon|+|\gamma|=|\alpha|}\left|D^{\varepsilon} \theta(x)\right|\left|D^{\gamma} f(x)\right| \leq c_{7}^{n^{2}} n^{c_{8} n^{2}} \rho^{-|\alpha|} \sup _{B(x, \rho)}|f|
$$

by Lemma 3.1 and Proposition 2.3. Here one has $\rho=\left(c_{3}^{n} n^{c_{4} n}\right)^{-1 / 2} \delta / \beta$, for $\delta\left(1-(10 \beta)^{-1}\right)<r<\delta$ and $\rho=\left(c_{3}^{n} n^{c_{4} n}\right)^{-1 / 2} h / \sqrt{\lambda}$, for $h / 2<r<2 h / 3$. Lemma 3.2 follows easily.

Let $\bar{r}$ be the conformally modified geodesic distance of Aronszajn $[1,3]$. This distance corresponds to a locally defined metric, of positive curvature, which is conformally equivalent to our original Riemannian metric. Given $\varepsilon>0$, one has $(1-\varepsilon) r<\bar{r}<(1+\varepsilon) r$, in a sufficiently small coordinate chart. By an easy induction argument, from the Carleman inequality in [3], one deduces

Proposition 3.3. Suppose that $u \in C_{0}^{\infty}$ is supported in $\delta / 2<r<h$. Then, for $\beta>a_{1} \sqrt{\lambda}+a_{2}$,

$$
\begin{aligned}
\iint \bar{r}^{2(2-\beta)}\left|\left(\prod_{i=1}^{n}\left(\Delta+\lambda_{i}\right)\right) u\right|^{2} \frac{d r d t}{r} \geq & b_{1}^{n} \beta^{2 n} \iint \bar{r}^{2(2-\beta)-2 n} u^{2} \frac{d r d t}{r} \\
& +b_{2}^{n} \delta^{n} \beta^{2 n} \iint \bar{r}^{2(2-\beta)-5 n} u^{2} \frac{d r d t}{r} .
\end{aligned}
$$

We proceed by substituting $u=\theta f$ into the Carleman inequality of Proposition 3.3. Using the estimates of Lemma 3.2 and the fact that $\left(\prod_{i=1}^{n}\left(\Delta+\lambda_{i}\right)\right) f=$ 0 , we find

$$
\begin{aligned}
\beta^{-1} \delta^{4-2 \beta}\left(\frac{\delta}{\beta}\right)^{-4 n} b_{3}^{n^{2}} n^{b_{4} n^{2}} \sup _{(1-1 / \beta) \delta \leq \bar{r} \leq(1+1 / \beta) \delta}|f|^{2} \\
+\left(\frac{h}{2}\right)^{4-2 \beta} h^{-4 n} b_{3}^{n^{2}} n^{b_{4} n^{2}} \lambda^{2 n} \sup _{h / 4 \leq \bar{r} \leq 3 h / 4}|f|^{2} \\
\geq b_{5}^{-n} \beta^{2 n}\left(\frac{h}{3}\right)^{2(2-\beta)-2 n} n^{-b_{6} n} \lambda^{-d / 2} \sup _{h / 12 \leq \bar{r} \leq h / 4}|f|^{2} \\
+b_{7}^{n} \delta^{n} \beta^{2 n} \iint_{\delta<\bar{r}<h / 2} \bar{r}^{2(2-\beta)-5 n}|f|^{2} r^{-1} d r d t .
\end{aligned}
$$


To absorb the last term on the left into the first term on the right, choose a parameter

$$
\beta>a_{1} \sqrt{\lambda}+a_{2}+a_{3} n^{2} \log n+a_{4} \log _{+}\left(\sup _{h / 4 \leq \bar{r} \leq 3 h / 4}|f| / \sup _{h / 12 \leq \bar{r} \leq h / 4}|f|\right) .
$$

This gives

$$
\begin{aligned}
\beta^{-1} \delta^{4-2 \beta}\left(\frac{\delta}{\beta}\right)^{-4 n} b_{3}^{n^{2}} n^{b_{4} n^{2}} \sup _{(1-1 / \beta) \delta \leq \bar{r} \leq(1+1 / \beta) \delta}|f|^{2} \\
\geq \\
\frac{1}{2} b_{5}^{-n} \beta^{2 n}\left(\frac{h}{3}\right)^{2(2-\beta)-2 n} n^{-b_{6} n} \lambda^{-d / 2} \sup _{h / 12 \leq \bar{r} \leq h / 4}|f|^{2} \\
+b_{7}^{n} \delta^{n} \beta^{2 n} \iint_{\delta<\bar{r}<h / 2} \bar{r}^{2(2-\beta)-5 n}|f|^{2} r^{-1} d r d t .
\end{aligned}
$$

We have now generalized the main technical estimate of [3]. Consequently, it is a routine matter to extend the discussion, by straightforward modifications of [3], to deduce

Corollary 3.4. (i) $\max _{\delta / 2 \leq \bar{r} \leq 3 \delta / 2}|f|^{2} \geq b_{8} \delta^{b_{9} \beta} \max _{h / 12 \leq \bar{r} \leq h / 4}|f|^{2}$,

(ii) $\max _{\bar{r} \leq 2 \delta}|f|^{2} \leq e^{b_{10} \beta} \max _{\bar{r} \leq \delta}|f|^{2}$,

(iii) $\max _{\bar{r} \leq \delta}|\nabla f| \leq b_{11}^{n} n^{b_{12} n^{2}} \beta^{(2 n+d+1) / 2} \delta^{-1} \max _{\bar{r} \leq \delta}|f|$.

\section{EIGENFUNCTIONS ON COMPACT MANIFOLDS}

Let $M$ be a compact Riemannian manifold. Assume that $f=\sum_{i=1}^{n} \phi_{i}$ is a finite sum of eigenfunctions, of the Laplacian $\Delta$, corresponding to distinct eigenvalues $-\lambda_{i} \geq-\lambda$. Our results on local properties of sums of eigenfunctions may be readily globalized, by analogy with the arguments from [3]. This gives

Theorem 4.1. (i) $f$ vanishes to at most order $\gamma$,

(ii) $\max _{B(x, 2 \delta)}|f| \leq e^{\gamma} \max _{B(x, \delta)}|f|$.

Here $\gamma=a_{1} \sqrt{\lambda}+a_{2} n^{2} \log n+a_{3}$. If the metric is real analytic, both $n$ and $\lambda$ may become arbitrarily large. For $C^{\infty}$ metrics, $\lambda$ may still be unbounded, but $n$ must remain less than a predetermined constant, $n \leq a_{4}$, as $\lambda \rightarrow \infty$.

Define the nodal set of $f$ to be $N=\{x \in M \mid f(x)=0\}$. If $d$ is the dimension of $M$, then let $\mathscr{H}^{d-1}$ denote the $(d-1)$-dimensional Hausdorff measure. The growth estimate of Theorem 4.1(ii), along with the techniques of [3], yield

Theorem 4.2. If $M$ is real analytic, then $\mathscr{H}^{d-1} N \leq c_{1}^{n} n^{c_{2} n} \sqrt{\lambda}$.

The dependence upon $n$ is worse than one might anticipate from inspection of Theorem 4.1 only. It occurs because we need to invoke the proof of analytic hypoellipticity, on scale $\rho=c_{3}^{-n} n^{-c_{4} n} \lambda^{-1 / 2}$ to obtain the bound

$$
\sup _{|z| \leq c_{s} \rho}|f(z)| \leq c_{6} \sup _{|x| \leq c_{7} \rho}|f(x)|
$$

for the holomorphic extension of $f$, where $z=\left(z_{1}, \ldots, z_{n}\right)$ lies in the complexification of a local coordinate path $x=\left(x_{1}, \ldots, x_{n}\right)$. Since $f$ need not vanish, a lower bound for the Hausdorff measure of $N$ seems problematic. 


\section{BIBLIOGRAPHY}

1. N. Aronszajn, A unique continuation theorem for solutions of elliptic partial differential equations of second order, J. Math. Pures Appl. 36 (1957), 235-249.

2. H. Cordes, Über die eindeutige Bestimmtheit Lösungen elliptischer Differentialgleichungen durch Anfangsvorgaben, Nachr. Akad. Wiss. Göttingen Math.-Phys. Kl. II 11 (1956), 239-258.

3. H. Donnelly and C. Fefferman, Nodal sets of eigenfunctions on Riemannian manifolds, Invent. Math. 93 (1988), 161-183.

4. L. Hörmander, Linear partial differential operators, Springer-Verlag, Berlin, Heidelberg, and New York, 1963.

Department of Mathematics, Purdue University, West Lafayette, Indiana 47907

E-mail address: hgdomath.purdue.edu 